\title{
Hepatocellular carcinoma: Consensus, controversies and future directions: A report from the Canadian Association for the Study of the Liver Hepatocellular Carcinoma Meeting
}

\author{
Kelly Warren Burak MD FRC-PC MSc(Epid) ${ }^{1}$, Morris Sherman MB BCh PhD FRC-PC ${ }^{2}$
}

\begin{abstract}
KW Burak, M Sherman. Hepatocellular carcinoma: Consensus, controversies and future directions: A report from the Canadian Association for the Study of the Liver Hepatocellular Carcinoma Meeting. Can J Gastroenterol Hepatol 2015;29(4):178-184.

Hepatocellular carcinoma (HCC) is a leading cause of cancer-related mortality worldwide and its incidence has rapidly increased in North America in recent years. Although there are many published guidelines to assist the clinician, there remain gaps in knowledge and areas of controversy surrounding the diagnosis and management of HCC. In February 2014, the Canadian Association for the Study of the Liver organized a one-day single-topic consensus conference on HCC. Herein, the authors present a summary of the topics covered and the result of voting on consensus statements presented at this meeting.
\end{abstract}

Key Words: Diagnosis; Hepatocellular carcinoma; Liver cancer; Practice guidelines; Surveillance; Therapy

\section{METHODOLOGY}

The present document is a report of the consensus conference on the management of hepatocellular carcinoma (HCC) sponsored by the Canadian Association for Study of the Liver (CASL), which was held in Toronto on February 11, 2014. This is not a practice guideline; however, the views expressed are those of physician experts on various aspects of the management of HCC. Where there is existing consensus, optimal management strategies are recommended, but we also sought to review controversial areas and define future direction in the management of HCC. The topics for the meeting were chosen by the organizing committee (KWB and MS), and nationally and internationally recognized experts in all aspects of HCC were invited to present (Table 1). The meeting was funded by the Canadian Liver Foundation and was endorsed by the World Gastroenterology Organization and the International Association for the Study of the Liver.

Before the meeting, several statements were drafted by the organizers related to each specific topic. The level of evidence for each statement was assigned according to the Oxford System (Table 2). Individual speakers first received the particular statements related to their specific topics for feedback and modification. After editing, the full set of statements was circulated to the entire group of speakers for comments and suggested changes. The finalized statements were circulated to all speakers and other participants ahead of the meeting, along with an environmental scan of the existing HCC consensus recommendations from the Canadian Multidisciplinary HCC Consensus and the major hepatology societies globally including the American Association for the Study of Liver Diseases (AASLD), the European Association for the Study of the Liver (EASL) and the Asian Pacific Association for the Study of the Liver (APASL) (1-4). Speakers delivered $20 \mathrm{~min}$ presentations on their topic, which are available at

\begin{abstract}
Le carcinome hépatocellulaire : consensus, controverses et futures orientations - rapport de la conférence sur le carcinome hépatocellulaire de l'Association canadienne pour l'étude du foie
\end{abstract}

Le carcinome hépatocellulaire (CHC) est une cause majeure de mortalité liée au cancer dans le monde. Ces dernières années, son incidence a augmenté rapidement en Amérique du Nord. Même si de nombreuses directives sont publiées pour venir en aide au clinicien, il reste des lacunes et des controverses à l'égard du diagnostic et de la prise en charge du CHC. En février 2014, l'Association canadienne pour l'étude du foie a organisé une conférence consensuelle d'une journée entièrement consacrée au CHC. Dans le présent article, les auteurs présentent un résumé des sujets traités et les résultats des votes sur les déclarations consensuelles qui y ont été présentées.

\footnotetext{
${ }^{1}$ Departments of Medicine and Oncology, Division of Gastroenterology $\mathcal{E}$ Hepatology, Cumming School of Medicine, University of Calgary, Calgary, Alberta; ${ }^{2}$ Department of Medicine, Division of Gastroenterology, University of Toronto and University Health Network, Toronto, Ontario Correspondence: Dr Kelly Warren Burak, Rm 6D35 Teaching Research and Wellness Building, 3280 Hospital Drive Northwest, Calgary, Alberta T2N 426.

Telephone 403-592-5049, fax 403-592-5090, e-mail kwburak@ucalgary.ca

Received for publication January 22, 2015. Accepted January 25, 2015
}

<www.hepatology.ca/?page_id=542>. There was a question and answer panel discussion following each section of related presentations, after which the participants in the audience voted on their level of agreement with the corresponding statements according to a five-point Likert scale (Table 3). The meeting was attended by 65 individuals, of whom 26 voted on the statements. The profession, years in practice and volume of HCC seen by the respondents were recorded (Figure 1). The majority were hepatologists and practicing physicians, with two being from nursing. The current report presents a summary of the topics presented and the results of the voting. We highlight areas in which there appears to be a lack of consensus regarding the diagnosis and management of HCC.

Epidemiology and surveillance

Incidence of HCC in Canada: The incidence of HCC in Canada in 2013 was 6.8 per 100,000 for men and 2.0 per 100,000 for women according to data collected by Statistics Canada (5). However, the incidence has been rising and has tripled in men and doubled in women since 1970 (6). Furthermore, there are reasons to suspect that the reported rates of HCC in Canada are underestimated. First, Statistics Canada collects data on primary liver cancers as HCC, cholangiocarcinoma, 'unspecified' and several other minor categories. The 'unspecified' category is approximately two-thirds of the total. In practice, it is rare to actually encounter a primary liver cancer that cannot be classified and, because HCC is, by far, the most common primary liver cancer, most of the 'unspecified' group is probably also HCC. Second, the number of cholangiocarcinomas is far too large as a proportion of all primary cancers, and many of these also likely represent HCC. More accurate data regarding the incidence and mortality due to HCC in Canada are needed. 
TABLE 1

Sessions, topics and speakers for the Canadian Association for the Study of the Liver hepatocellular carcinoma meeting

\begin{tabular}{|c|c|c|c|}
\hline Session & Topic & Speaker & Institution \\
\hline \multirow{3}{*}{$\begin{array}{l}\text { Epidemiology and } \\
\text { surveillance }\end{array}$} & Hepatocellular carcinoma in Canada & Morris Sherman & University of Toronto (Toronto, Ontario) \\
\hline & Surveillance: Evidence and populations & Jordan Feld & University of Toronto (Toronto, Ontario) \\
\hline & Surveillance: Tests, interval, uptake & Amit Singal & University of Texas (Austin, Texas, USA) \\
\hline \multirow[t]{4}{*}{ Diagnosis } & Very early stage hepatocellular carcinoma & Morris Sherman & University of Toronto (Toronto, Ontario) \\
\hline & $\begin{array}{l}\text { Computed tomography, magnetic resonance imaging, } \\
\text { Li-RADS }\end{array}$ & An Tang & Université de Montreal (Montreal, Quebec) \\
\hline & Contrast-enhanced ultrasound & Stephanie Wilson & University of Calgary (Calgary, Alberta) \\
\hline & Gd-EOB-DTPA magnetic resonance imaging & Tae Kyoung Kim & University of Toronto (Toronto, Ontario) \\
\hline \multirow[t]{2}{*}{ Staging and prognosis } & Barcelona Clinic Liver Cancer system & Jordi Bruix & University of Barcelona (Barcelona, Spain) \\
\hline & Predicting recurrence & Peter Ghali & McGill University (Montreal, Quebec) \\
\hline \multirow{5}{*}{$\begin{array}{l}\text { Current curative and } \\
\text { palliative therapies }\end{array}$} & Surgery & Sean Cleary & University of Toronto (Toronto, Ontario) \\
\hline & Radiofrequency ablation/percutaneous ethanol injection & John Kachura & University of Toronto (Toronto, Ontario) \\
\hline & Transplantation & Norman Kneteman & University of Alberta (Edmonton, Alberta) \\
\hline & Transarterial chemoembolization & Kelly Burak & University of Calgary (Calgary, Alberta) \\
\hline & Sorafenib & Andrew Zhu & Harvard University (Boston, Massachusetts, USA) \\
\hline \multirow{3}{*}{$\begin{array}{l}\text { Experimental therapy } \\
\text { and future directions }\end{array}$} & Transarterial radioembolization & Aldo Montano-Loza & University of Alberta (Edmonton, Alberta) \\
\hline & Stereotactic body radiation treatment & Laura Dawson & University of Toronto (Toronto, Ontario) \\
\hline & New drugs for hepatocellular carcinoma & Andrew Zhu & Harvard University (Boston, Massachusetts, USA) \\
\hline
\end{tabular}

Gd-EOB-DTPA Gadolinium ethoxybenzyl diethylenetriamine penta-acetic acid; Li-RADS Liver imaging reporting and data system

TABLE 2

Oxford system for grading level of evidence

\begin{tabular}{ll}
\hline Level & Type of evidence \\
\hline AA & Systematic review of randomized clinical trials \\
1C & Individual RCTs with narrow confidence intervals \\
$2 \mathrm{~A}$ & All or none studies \\
2B & Systematic reviews of cohort studies \\
2C & Individual cohort study including low-quality RCTs \\
$3 \mathrm{~A}$ & Outcomes research; ecological studies \\
$3 \mathrm{~B}$ & Systematic review of case-control studies \\
4 & Individual case-control studies \\
5 & Case-series and poor quality cohort and case-control studies \\
& Expert opinion without explicit critical appraisal or descriptive \\
\hline
\end{tabular}

RCTs Randomized controlled trials

TABLE 3

Likert scale used to assess degree of consensus to statements

\begin{tabular}{lllll}
\hline $\mathbf{1}$ & $\mathbf{2}$ & $\mathbf{3}$ & $\mathbf{4}$ & $\mathbf{5}$ \\
\hline Accept & Accept with & Accept with & Reject with & Reject \\
completely & some & major & some & completely \\
& reservation & reservation & reservation & \\
\hline
\end{tabular}

Modelling studies for both hepatitis B virus (HBV) and hepatitis C virus suggest that the incidence of HCC will continue to rise for the next 15 to 20 years $(7,8)$. Furthermore, as the obesity and diabetes epidemics continue to increase we are also likely to encounter more HCC related to nonalcoholic fatty liver disease. The incidence of HCC will, therefore, likely continue to climb for years to come.

Surveillance: Evidence and populations: The evidence for HCC surveillance is supported by case-control and cohort studies, as well as one randomized controlled trial (RCT) from China involving HBVinfected individuals (9). The strength of evidence around HCC surveillance has been criticized (10); however, further RCTs are not likely to be performed. There is evidence that surveillance for HCC is cost effective, but this is highly dependent on incidence of HCC in the population under surveillance. The groups who should be offered surveillance have been previously identified (1-3). The categories of

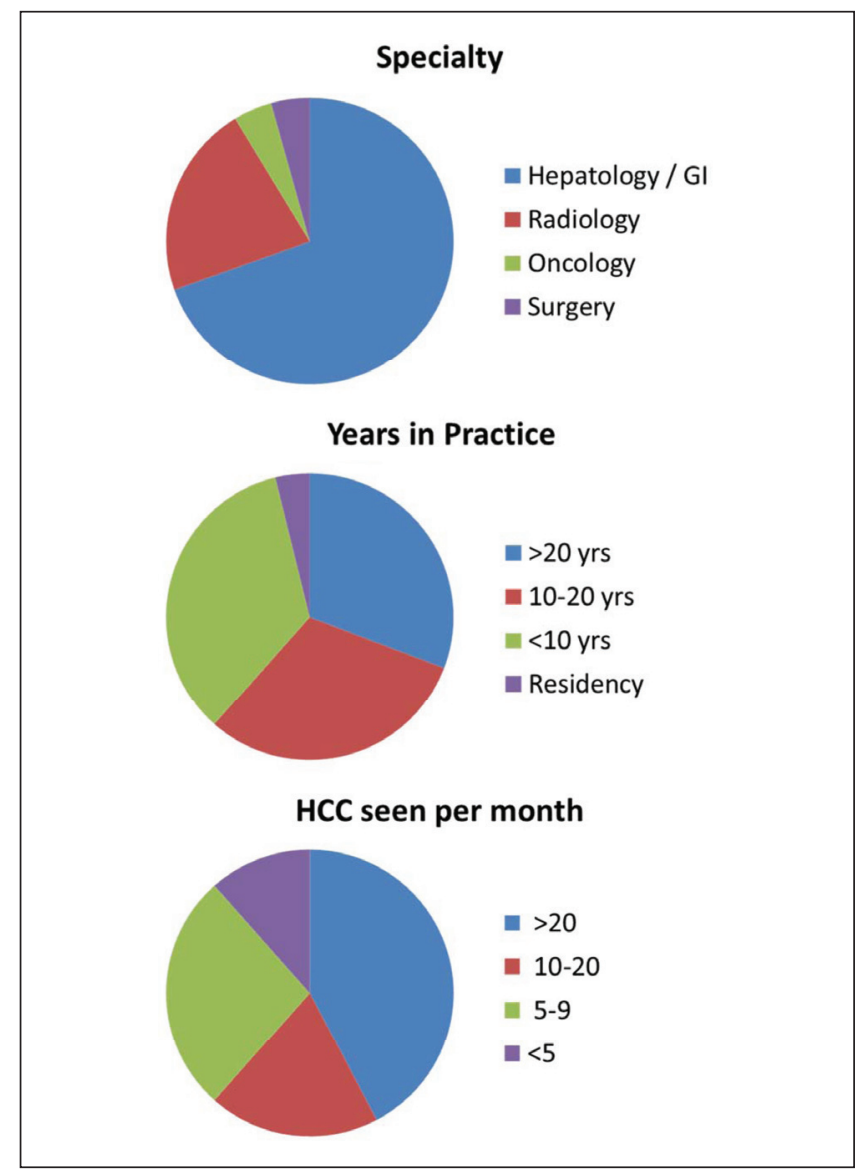

Figure 1) Demographics of voters. GI Gastroenterology; HCC Hepatocellular carcinoma; yrs Years

patients are broadly defined as all cirrhotic and certain noncirrhotic patients with HBV (Asian men $>40$, Asian women $>50$ and Africans $>20$ years of age, positive family history) (1-3). However, surveillance should not be offered to Child-Pugh (C-P) class $\mathrm{C}$ cirrhotic patients, unless they are awaiting liver transplantation (LT) (1-3). A large 


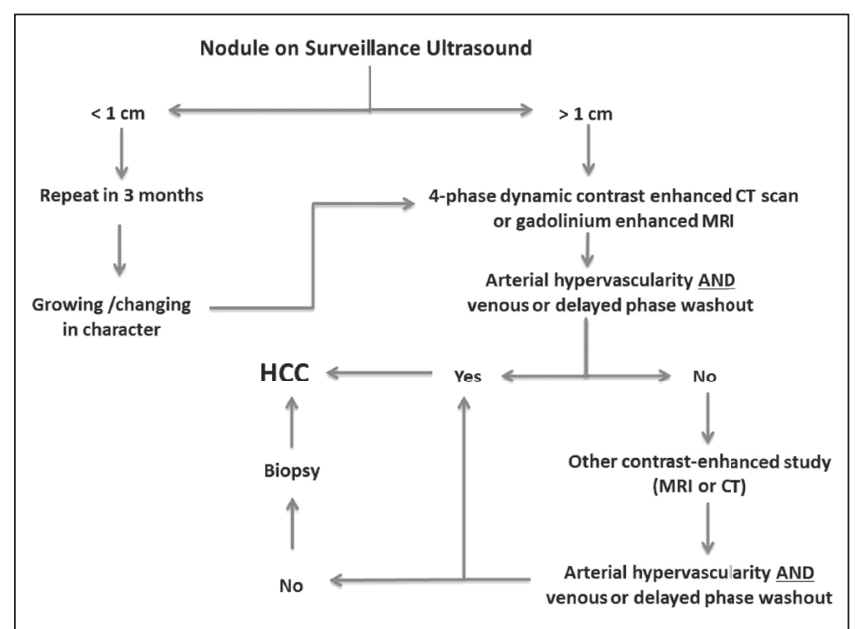

Figure 2) Algorithm for the diagnosis of hepatocellular carcinoma (HCC) after identification of a lesion on a surveillance ultrasound. CT Computed tomography; MRI Magnetic resonance imaging. Adapted with permission from reference 2

cohort study of ambulatory cirrhotic patients from the University of Toronto (Toronto, Ontario) has shown that the risk of HCC is highest for viral hepatitis, followed by steatohepatitis and other causes of cirrhosis (11). There are other risk scores for HBV and for cirrhotic patients, and these may be used to help identify those at highest risk for HCC (12). These risk scores require further validation before being routinely used to determine who should undergo surveillance and those for whom surveillance is unnecessary.

Surveillance: Tests, intervals and uptake: Ultrasound (US) is effective for HCC detection with sensitivity of approximately 63\%, and six months is superior to annual surveillance (13). However, US has limitations in clinical practice and can be influenced by the experience and training of technicians, and the presence of obesity, cirrhosis and/or ascites. In general, surveillance is underutilized, with $<20 \%$ of cirrhotic patients receiving regular surveillance (14).

The use of biomarkers, such as alpha-fetoprotein (AFP), AFP-L3 or des-gamma-carboxy prothrombin, is controversial. AFP can be combined with US to maximize early tumour detection; however, this may be associated with higher false-positive rates and costs (15). Evidence also suggests that these biomarkers are not highly sensitive to the presence of small HCCs, and are more frequently associated with aggressive or late-stage disease, making these markers unsuitable for HCC surveillance. Better biomarkers are clearly required for HCC surveillance. Statements on surveillance:

- Surveillance for HCC is cost effective in high-risk groups (Level 2C); however, surveillance should only be offered to patients who are candidates for therapy (Level 5). Mean rating $1.27(1=19,2=7,3=0,4=0,5=0)$.

- The risk for HCC is highest in cirrhotic patients with viral hepatitis (HBV or hepatitis $\mathrm{C}$ virus) followed by those with steatohepatitis (alcohol or nonalcoholic) (Level 2B). Mean rating $1.46(1=17,2=8,3=0,4=0,5=1)$.

- Surveillance should be offered to all patients with cirrhosis and certain chronic carriers of HBV without established cirrhosis (Level 2B). Mean rating $1.38(1=17,2=8,3=1,4=0,5=0)$.

- Risk scores may help better identify high-risk groups (Level 2C), but their routine use requires further validation. Mean rating $1.46(1=16,2=9,3=0,4=1,5=0)$.

- Surveillance should be performed using US every six months (Level 1B), performed by experienced ultrasonographers (Level 5). Mean rating $1.08(1=25,2=0,3=1,4=0,5=0)$.

- AFP alone should not be used for surveillance (Level 2B). AFP when combined with US will detect more HCC but increases the cost of surveillance due to investigations of false positives and is more likely to detect $\mathrm{HCC}$ at a later stage (Level 2B).

Mean rating $1.58(1=15,2=8,3=2,4=1,5=0)$.

- Other biomarkers (eg, AFP-L3 and des-gamma-carboxy prothrombin) may be associated with more advanced stage of HCC (Level 2B); therefore, their role in surveillance requires further study. Mean rating $1.62(1=13,2=10,3=3,4=0,5=0)$.

\section{Diagnosis}

Very early stage HCC: The diagnosis of HCC can be established with radiology or by biopsy. The classical radiographic appearance of HCC is a lesion that exhibits higher signal intensity than the surrounding liver in the arterial phase of a contrast-enhanced study and lower signal intensity than the surrounding liver in the venous or the delayed phase of the contrast examination (so-called portal venous 'washout'). The mechanism underlying this phenomenon is that the HCC is a vascular lesion, fed principally by the hepatic artery, whereas the liver receives $80 \%$ of its blood supply from the portal vein. However, some HCCs are hypovascular, particularly if small or early. In these lesions, the arterial supply may not be fully developed, and they may present as hypovascular lesions on dynamic imaging (16).

If the radiological appearances are not typical a biopsy is required. Most often in larger lesions the interpretation of the biopsy is not difficult, but with smaller lesions the distinction between dysplastic nodule and HCC becomes more difficult. In addition to cellular features, a set of stains can reliably separate lesions into HCC or not HCC. These include glypican 3, heat shock protein 70 , glutamine synthetase and clathrin heavy chain. The likelihood of HCC approaches 100\% when at least two of these markers are positive (17), and these stains should be used in all cases in which the diagnosis is in doubt.

Computed tomography, magnetic resonance imaging, liver imaging reporting and data system: A diagnostic algorithm for HCC has been developed and validated (Figure 2) (2). For any lesion $>1 \mathrm{~cm}$ found on surveillance US, the diagnosis of HCC can be made if either the contrast-enhanced computed tomography (CT) scan or magnetic reonance imaging (MRI) show typical features as described above. If there is no arterial phase enhancement or no portal venous or delayed phase washout, and if the radiological appearances are not typical of another type of lesion (eg, hemangioma), a biopsy is required. All major clinical practice guidelines endorse multiphasic CT and MRI with extracellular contrast agents as the first-line modalities for diagnosis and staging of HCC (1-4).

The American College of Radiology has developed liver imaging reporting and data system (LI-RADS) as a standardized system for interpretation and data collection of CT and MRI liver examinations (18). LI-RADS categorize observations along a five-point ordinal scale ranging from LR1 (definitely benign) to LR5 (definitely HCC). The application of LI-RADS has yet to be validated; however, standardized reporting should improve communication between radiologists and other clinicians caring for HCC patients.

Contrast-enhanced US: The APASL guidelines state that contrastenhanced US (CEUS) is as sensitive as dynamic CT or dynamic MRI in the diagnosis of HCC (4); however, the EASL (3) and AASLD (2) guidelines have dropped CEUS from the diagnostic algorithm (Figure 2) because some cholangiocarcinomas on CEUS can have a pattern of arterial phase enhancement and portal venous washout similar to HCC (19). It is important to recognize that CEUS remains a valuable tool in the diagnosis and management of HCC. Unlike dynamic CT or MRI, CEUS allows for real-time scanning throughout the entire arterial phase, with a contrast agent that is purely intravascular and can be safely administered in patients with renal failure. In fact, very rapid washout on CEUS is atypical for HCC and is more likely to represent cholangiocarcinoma or metastatic disease. Furthermore, CEUS can assist in the staging of HCC by determining whether portal vein thrombosis (PVT) is bland or malignant, and may be very helpful in localizing and assessing response of small HCC undergoing percutaneous ablation (20). 
Gd-EOB-DPTA MRI: Gadolinium ethoxybenzyl diethylenetriamine penta-acetic acid (Gd-EOB-DTPA) is a liver-specific contrast agent that has been evaluated in the diagnosis of liver lesions, including HCC (21). It is more sensitive than CT scan for detecting HCC. However, it is more expensive than regular gadolinium contrast, has weaker arterial phase enhancement, and requires a delayed phase (usually performed at $20 \mathrm{~min}$ ). Nodules without classic features for HCC on dynamic MRI, that remain hypointense on the delayed phase following administration of Gd-EOB-DPTA, may represent HCC. Whether it should used as the sole diagnostic agent in all cases or only used in specific cases of diagnostic difficulty has yet to be clarified.

\section{Statements on diagnosis:}

- In cirrhotic patients, a noninvasive diagnosis of HCC can be established for a lesion $>1 \mathrm{~cm}$ if contrast-enhanced imaging (CT, MRI or CEUS) demonstrates hypervascularity in the arterial phase with washout in the portal venous phase (Level 2B). Mean rating $1.28(1=18,2=7,3=0,4=0,5=0)$.

- The pathological diagnosis of HCC should follow the recommendations of the International Consensus Panel (Level 2B). Immunostaining is recommended to help differentiate highgrade dysplastic nodules from early HCC (Level $2 \mathrm{~B})$. Mean rating $1.5(1=15,2=6,3=3,4=0,5=0)$.

- Patients at risk for HCC with a lesion $>1 \mathrm{~cm}$ detected on US should undergo a contrast-enhanced CT or contrast-enhanced MRI to establish the diagnosis of HCC and stage the disease (Level 2A). Mean rating $1.19(1=23,2=1,3=2,4=0,5=0)$.

- LI-RADS is recommended to standardize the reporting of CT and MRI in patients with cirrhosis or other risk factors for HCC because it enables consistent terminology, reduced interpretation variability, enhanced communication with clinicians and facilitates quality assurance and research.

Mean rating $2.12(1=11,2=7,3=3,4=4,5=1)$.

- CEUS demonstrating arterial phase enhancement and late phase washout can be used for the diagnosis HCC (Level 2B). Mean rating $1.54(1=16,2=8,3=1,4=0,5=1)$.

- CEUS is particularly useful for evaluation of venous thrombosis (bland versus malignant), for directing radiofrequency ablation (RFA) of small HCC and for surveillance of recurrence following curative intent therapy (Level 5). Mean rating $1.62(1=13,2=10$, $3=3,4=0,5=0$ ).

- Although Gd-EOB-DTPA-enhanced MRI yields higher diagnostic accuracy compared with four-phase CT, its role in the management of $\mathrm{HCC}$ is not yet defined (Level $2 \mathrm{~B}$ ). Mean rating $1.48(1=17,2=5,3=2,4=1,5=0)$.

- Nodules with atypical enhancement on the dynamic phases but with hypo-enhancement on the hepatobiliary phase of Gd-EOBDTPA-enhanced MRI may represent HCC. Close surveillance or biopsy of these lesions is recommended (Level 5). Mean rating $1.62(1=15,2=8,3=2,4=0,5=1)$.

\section{Staging and prognosis}

Barcelona Clinic Liver Cancer System: There are several ways to assess the severity of disease in patients with HCC. Liver function can be assessed by the C-P score or the Model for End-stage Liver Disease score. The TNM (tumour-node-metastasis) system assesses the anatomical extent of the cancer. Performance scores such as the Karnovsky or the Eastern Cooperative Oncology Group performance status assess the functional limitations of the patient due to the cancer. However, none of these are adequate on their own for HCC, and none give any guidance as to treatment. There are several HCC staging systems that attempt to take some of these factors into account, but the only the Barcelona Clinic Liver Cancer (BCLC) system uses performance status, liver function and tumour burden together to recommend specific therapies. Furthermore, the BCLC staging system has been validated externally for predicting prognosis. The current iteration of the BCLC staging system is shown in Figure 3 (22).

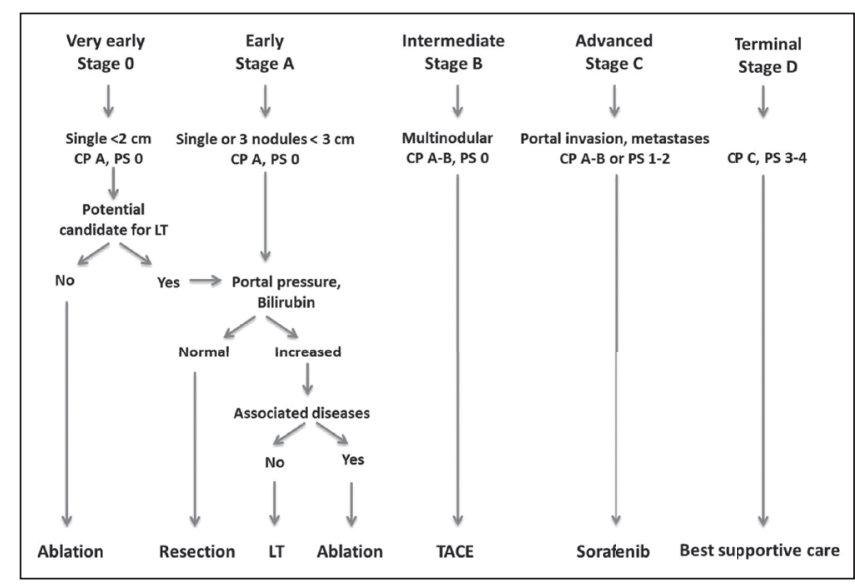

Figure 3) Barcelona Clinic Liver Cancer stages and allocation of treatment. CP Child-Pugh class; LT Liver transplantation; PS Eastern Cooperative Oncology Group performance status; TACE Transarterial chemoembolization. Adapted with permission from reference 22

Some have suggested that the intermediate stage (BCLC B) could be further stratified into four categories (B1 to B4), but this needs external validation (23).

Predicting recurrence: Within five years of curative therapy, recurrence of HCC occurs in approximately $70 \%$ of patients treated with surgery or RFA compared with $<15 \%$ of patients undergoing LT. Size and number of tumours predict vascular invasion and subsequent recurrence after LT, and thereby form the basis of the Milan criteria (single tumour $\leq 5 \mathrm{~cm}$, or $\leq 3$ lesions each $\leq 3 \mathrm{~cm}$ ) (24). AFP has been shown to predict recurrence after RFA and resection. Furthermore, a high AFP, especially if climbing, predicts recurrence after LT. In the near future, both Canada and the United States will be adopting an AFP $<400 \mathrm{ng} / \mathrm{mL}$ as a listing requirement for LT. In the future, genomic signatures of the tumour and surrounding liver may help provide further prognostication about risk of recurrence (25).

Statements on staging and prognosis:

- The BCLC staging system is preferred because it takes into account the extent of tumour burden, the function of the liver (C-P score), and the Eastern Cooperative Oncology Group performance status of the patient while recommending evidence based treatment for specific stages (Level 2A). Mean rating $1.4(1=18,2=5,3=1,4=1,5=0)$.

- Patients diagnosed with HCC should have an AFP level performed because serial measurements of AFP can provide additional prognostic information (Level 2A). Mean rating $1.74(1=12,2=7,3=3,4=0,5=1)$.

- Enhanced surveillance for HCC recurrence is required in patients undergoing surgery or RFA because recurrence occurs in up to $70 \%$ of patients within five years (Level 2A). Mean rating 1.24 $(1=21,2=2,3=2,4=0,5=0)$.

- Adjuvant therapy after surgery, RFA or LT is not recommended (Level 2B); however, treatment of viral hepatitis should be considered to lower the risk of HCC recurrence (Level 2B). Mean rating $1.42(1=16,2=6,3=2,4=0,5=0)$.

\section{Current curative therapies}

The therapies that are considered to be curative include resection, local ablation with RFA, microwave ablation or percutaneous ethanol injection (PEI) for small lesions, and LT.

Resection: Whether resection is possible depends largely on liver function. Patients should have C-P class A cirrhosis, no significant portal hypertension and a normal serum bilirubin level (26). In addition, the amount of liver to be removed and the volume of the remaining liver are important. Anatomical resection refers to removal of one or more segments, and is always preferable because disease-free and overall survival are improved (27). Hepatic resection can be 
accomplished by laparoscopy with considerable reduction in morbidity, but requires adequate training.

RFA/PEI: RFA can be suitable for patients with small lesions (ideally $<3 \mathrm{~cm}$ ) who have liver function that does not allow resection. RFA can also be used for local control of the HCC as bridging therapy before LT. RFA is clearly superior to PEI for lesions between $2 \mathrm{~cm}$ and $5 \mathrm{~cm}$ (28). Multifocal HCC is also associated with a higher rate of incomplete response to RFA.

A meta-analysis of RCTs and non-RCTs comparing RFA and resection found that RFA is better tolerated than resection, with similar short-term outcomes (29). Recurrence rates are higher with RFA, and this translates into lower overall survival at five years for RFA compared with surgery (29). However, for smaller lesions $(<2 \mathrm{~cm})$, recurrence rates and overall survival are comparable, making RFA an acceptable substitute for surgery in selected patients (29).

LT: LT has traditionally been limited to patients who fell within the 'Milan criteria', namely, one HCC lesion $<5 \mathrm{~cm}$ or fewer than three lesions, none larger than $3 \mathrm{~cm}$ in diameter (24). However, there are clearly patients within the Milan criteria who develop recurrence posttransplantation, and there are also many patients with HCC beyond the Milan criteria who can be cured by LT. There have been several proposals to extend these criteria, including the University of California San Francisco criteria, the "up to seven" criteria or total tumour volume (TTV). Any expansion of the listing criteria is associated with a higher drop-out rate on the LT waitlist and higher posttransplant recurrence rates. TTV $<115 \mathrm{~cm}^{3}$ and AFP $<400 \mathrm{ng} / \mathrm{mL}$ predict survival after LT, and they have been prospectively validated as selection criteria for LT in Canada (30).

Statements on curative therapies:

- Surgery is first-line therapy for patients with single (and selected multifocal) $\mathrm{HCC}$ who have well-preserved liver function (C-P class A) (Level 2A). Mean rating $2.0(1=8,2=11,3=4,4=2$, $5=0$ ).

- Important predictors for the function of the future liver remnant include portal hypertension (platelets $<100 \times 109 / \mathrm{L}$, presence of varices, or hepatic venous pressure gradient $>10 \mathrm{mmHg}$ ) and a normal serum bilirubin (Level 2B). Mean rating $1.2(1=20,2=5$, $3=0,4=0,5=0$ ).

- Anatomic resection should be performed where possible (Level 2B). Mean rating $1.25(1=19,2=4,3=1,4=0,5=0)$.

- Patients should be considered for laparoscopic hepatic resection where adequate technical training and experience exist (Level 5). Mean rating $1.64(1=13,2=10,3=1,4=0,5=1)$.

- The recurrence rates are lower following surgical resection of early stage HCC (within Milan criteria) than with RFA (Level 1B). Mean rating $2.08(1=9,2=7,3=5,4=3,5=0)$.

- Local ablation is recommended for early stage HCC (within Milan criteria) who are not candidates for surgical resection (Level 2A) and can be used to bridge patients to LT (Level 4). Mean rating $1.42(1=16,2=7,3=0,4=1,5=0)$.

- For BCLC stage 0 HCC $(\leq 2 \mathrm{~cm})$, ablation may be an effective and less-invasive alternative to surgical resection (Level 2B). Mean rating $1.16(1=21,2=4,3=0,4=0,5=0)$.

- RFA is preferred to PEI for lesions $2 \mathrm{~cm}$ to $5 \mathrm{~cm}$ in size (Level 1A). Mean rating $1.25(1=19,2=4,3=1,4=0,5=0)$.

- LT should be considered in patients HCC who have developed complications of cirrhosis (Level 5). Mean rating 1.18 $(1=19,2=2,3=1,4=0,5=0)$.

- LT is the first-line therapy for patients within the Milan criteria who are not candidates for resection or ablation (Level 2A). Mean rating $1.3(1=19,2=3,3=0,4=0,5=1)$.

- LT can be used to salvage patients with recurrence after resection or RFA (Level 4). Mean rating $1.52(1=14,2=6,3=3,4=0,5=0)$.

- Acceptable outcomes (five-year survival $>70 \%$ ) can be achieved in carefully selected patients beyond the Milan criteria (eg, TTV $<115 \mathrm{~cm}^{3}$ AND AFP $<400 \mathrm{ng} / \mathrm{mL}$ ); however, higher waitlist drop-out and recurrence rates following LT may be seen with extended criteria (Level 2B). Mean rating $1.57(1=12,2=9$, $3=2,4=0,5=0$ ).

\section{Current palliative therapies}

There are only two palliative forms of therapy that have been shown to improve survival in randomized clinical trials: transarterial chemoembolization (TACE) and sorafenib.

TACE: Conventional TACE for BCLC stage B HCC is associated with a median survival of approximately two years; unfortunately, the TACE procedure is not standardized (31). TACE has been used to keep patients within selection criteria while awaiting LT, although the exact impact of this on post-LT recurrence requires further study. TACE should be avoided in decompensated cirrhosis and patients with PVT.

A more recent innovation is TACE delivered by drug-eluting beads (DEBs). These have been compared head-to-head with conventional TACE, with no difference in survival, but less toxicity when using the DEBs (32). Cohort studies are now reporting fouryear median survival for TACE using doxorubicin-loaded DEBs in carefully selected patients (33). Combination of sorafenib and TACE does not offer a survival advantage.

Sorafenib: Sorafenib for BCLC stage C disease provides a median improvement in survival of approximately three months. This has been demonstrated in two large RCTs $(34,35)$. The survival benefit of sorafenib is only established in patients with preserved liver function (C-P class A cirrhosis). Sorafenib is generally well tolerated but is associated with hand/foot skin reaction, fatigue, gastrointestinal symptoms and hypertension. The standard dose is $400 \mathrm{mg}$ twice daily; however, dose escalation is frequently used in clinical practice. Sorafenib has no advantage when used as adjuvant treatment after any of resection, RFA or TACE.

\section{Statements on palliative therapies:}

- TACE is standard of care for patients with large single HCC who are not candidates for resection or ablation (BCLC stage A) or for multifocal HCC without evidence of portal vein invasion or extrahepatic spread (BCLC stage B) (Level 1A). Mean rating $1.27(1=17,2=4,3=1,4=0,5=0)$.

- TACE can be used to bridge patients to LT (Level 4). Mean rating $1.24(1=16,2=5,3=0,4=0,5=0)$.

- TACE should be avoided in patients with main PVT and in patients with decompensated cirrhosis (Level 5). Mean rating $1.05(1=20,2=1,3=0,4=0,5=0)$.

- Compared with conventional TACE, DEBs provide a more standardized technique with a better safety profile (Level 1B). Mean rating $1.36(1=14,2=8,3=0,4=0,5=0)$.

- Patients with disease progression after two TACE should be considered for sorafenib or clinical trials evaluating sorafenib in conjunction with transarterial radioembolization (TARE) or stereotactic body radiotherapy (SBRT) (Level 5). Mean rating $1.76(1=9,2=8,3=4,4=0,5=0)$.

- Sorafenib is first-line therapy for patients with advanced HCC due to portal vein invasion or metastatic disease (BCLC stage C), or in patients who have progressed after TACE (BCLC stage B) (Level 1B). Mean rating $1.55(1=13,2=8,3=4,4=0,5=1)$.

- Sorafenib should only be used in patients with preserved liver function (C-P class A) (Level 1B). Mean rating $1.77(1=8,2=11,3=3,4=0,5=0)$.

Experimental treatments and future directions Treatments are considered to be experimental if outcome data are lacking or if there is inadequate comparison with current standard treatments. This is includes TARE and SBRT.

TARE: TARE involves infusing radioactive particles, either glass beads or resin, via the hepatic artery directly into the tumour. This technique has been shown to produce substantial tumour necrosis, is 
safe, and can be administered to patients with PVT (36). TARE is well tolerated and has less impact on liver function than TACE. Although it has not been compared directly with TACE in a randomized trial, it does appear to have similar outcomes to TACE (37). In a single nonrandomized trial (38), TARE achieved better downstaging than TACE, but long-term post-LT outcomes were not reported. The addition of TARE to sorafenib for advanced-stage HCC is being evaluated in the ongoing phase III Efficacy Evaluation of TheraSphere in Patients With Inoperable Liver Cancer (STOP-HCC) clinical trial (NCT01556490).

SBRT: External beam radiotherapy is a relatively new form of therapy for HCC. Furthermore, stereotactic administration of radiotherapy limits toxicity to the liver and surrounding organs. Phase I and II trials have shown efficacy in achieving disease control; again, there has not been any direct comparison between radiotherapy and any other form of treatment (39). Radiotherapy has also been used to treat malignant portal vein thrombus and to bridge patients to LT. The addition of SBRT to sorafenib for $\mathrm{HCC}$ is being evaluated in the ongoing phase III Sorafenib Tosylate With or Without Stereotactic Body Radiation Therapy in Treating Patients With Liver Cancer (RTOG1112) clinical trial (NCT01730937).

Systemic chemotherapy: Systemic chemotherapy has not been shown to significantly enhance survival and, therefore, should not be used outside of clinical trials. Sorafenib with or without doxorubicin is currently being evaluated in the Sorafenib Tosylate With or Without Doxorubicin Hydrochloride in Treating Patients With Locally Advanced or Metastatic Liver Cancer (HEC.1) trial (NCT01015833). Future directions: Several targeted therapies, including sunitinib (40) and brivanib (41), have failed when compared with sorafenib in RCTs. The addition of erlotinib to sorafenib was not beneficial in advanced HCC (42). Brivanib (43) and everolimus (44) have recently failed to show benefit as second-line therapy after sorafenib. Currently, many drugs are under study for HCC; there remains an unmet need for new first- and second-line therapies (45).

Statements on experimental therapies:

- TARE may be considered if patients are poor TACE candidates (eg, PVT) (Level 2B). Mean rating $1.84(1=7,2=9,3=2,4=1$, $5=0$ ).

- TARE may be more likely to downstage T3 tumours compared with TACE (Level 3B). Mean rating $2.47(1=3,2=7,3=3,4=4$, $5=0$ ).

- Further RCTs evaluating TARE are needed to better define its role in HCC (Level 5). Mean rating $1.16(1=17,2=1,3=1,4=0$, $5=0$ ).

- SBRT may have a role in HCC management, including bridging some patients to LT (Level 4). Mean rating $1.78(1=9,2=5,3=3$, $4=1,5=0$ ).

- Further RCTs evaluating SBRT are needed to better define its role HCC (Level 5). Mean rating $1.22(1=16,2=1,3=0,4=1,5=0)$.

- Systemic chemotherapy (eg, doxorubicin) is not recommended outside of clinical trials (Level 2B). Mean rating 1.33 ( $1=15,2=1$, $3=1,4=1,5=0$ ).

- Further study into second-line agents for patients progressing on sorafenib are needed (Level 5). Mean rating $1.21(1=17,2=1$, $3=0,4=1,5=0)$.

\section{DISCUSSION}

Overall, there was good agreement on the consensus statements presented, with only four statements having a mean rating of $\geq 2.0$ on the five-point Likert scale. The first of these concerned LI-RADS and may reflect an unfamiliarity of clinicians with this new reporting system. LI-RADS is currently undergoing modifications and has not been widely implemented in Canada. However, it has recently been endorsed by the United Network of Organ Sharing and will be a requirement for reporting $\mathrm{CT}$ and MRI of HCC patients awaiting LT in the United States.
Second, several participants were not comfortable recommending surgery as the first-line therapy in HCC patients with C-P class A cirrhosis. This may be reflective of the fact that the majority of the audience consisted of hepatologists, who may be more comfortable arranging RFA or LT evaluation for these patients. Similarly, many disagreed with the statement that recurrence rates are lower following surgical resection of early stage HCC compared with RFA. Although this is Level $1 \mathrm{~B}$ evidence, many in the audience may have recognized the methodological flaws in the three RCTs from China on which this recommendation is based, including significant cross-over between groups after randomization.

Finally, there was significant reservation around a statement that TARE may be more likely to downstage large tumours compared with TACE. Again, there may be a lack of familiarity with TARE because this is only available in a few Canadian centres, or perhaps it is recognition that this recommendation is largely based on a single casecontrol study from Chicago (Illinois, USA).

\section{CONCLUSIONS}

HCC is rapidly increasing in Canada and its incidence is expected to continue to rise for years to come. Surveillance with US alone every six months is currently recommended for high-risk populations. The diagnosis of HCC can usually be achieved with CT or MRI, although CEUS and Gd-EOB-DTPA MRI may assist in the diagnosis in some cases. The management of HCC is complex and must be performed in a multidisciplinary team setting. The BCLC system is recommended for staging and for treatment allocation. In carefully selected patients, resection, RFA and LT can offer a cure, whereas TACE and sorafenib extend life but are palliative therapies. Further research is required on TARE and SBRT before they become standard of care. New, more-effective therapies are needed for advanced-stage HCC.

ACKNOWLEDGEMENTS: The authors thank the speakers (listed in Table 1) for their significant contributions to the meeting, especially $\mathrm{Dr}$ Jordi Bruix (CASL Gold Medal recipient) for his critical review and feedback on the consensus statements. We recognize the efforts of Allison Bollieu (CASL secretariat) and BUKSA for meeting organization, and thank Karen Seto and the Canadian Liver Foundation for their assistance in raising funds for this meeting through unrestricted educational grants.

COLLABORATORS: Jordan Feld, Amit Singal, An Tang, Stephanie Wilson, Tae Kyoung Kim, Jordi Bruix, Peter Ghali, Sean Cleary, John Kachura, Norman Kneteman, Andrew Zhu, Aldo Montano-Loza and Laura Dawson.

DISCLOSURES: This meeting was sponsored by the Canadian Liver Foundation and its industry partners. Kelly W Burak has received research support (Bayer, Oncozyme) and honoraria (Astellas, Gilead) within the last year. Morris Sherman has received honoria within the last year from Janssen Canada, Merck, Gilead, Oncozyme, AASLD and WAKO.

\section{REFERENCES}

1. Sherman M, Burak K, Maroun J, et al. Multidisciplinary Canadian consensus recommendations for the management and treatment of hepatocellular carcinoma. Curr Oncol 2011;18:228-40.

2. Bruix J, Sherman M; American Association for the Study of Liver Diseases. Management of hepatocellular carcinoma: An update. Hepatology 2011;53:1020-2.

3. European Association For The Study Of The Liver; European Organization For Research And Treatment Of Cancer. EASLEORTC clinical practice guidelines: Management of hepatocellular carcinoma. J Hepatol 2012;56:908-43.

4. Omata M, Lesmana LA, Tateishi R, et al. Asian Pacific Association for the Study of the Liver consensus recommendations on hepatocellular carcinoma. Hepatol Int 2010;4:439-74.

5. Public Health Agency of Canada Mortality Statistics. < www.phacaspc.gc.ca/sti-its-surv-epi/model/pdf/model07-eng.pdf $>$ (Accessed February 1, 2014). 
6. Canadian Cancer Statistics 2013. Special Report: Liver Cancer. $<$ www.cancer.ca/en/cancer-information/cancer-101/canadiancancer-statistics-publication/past-editions-canadian-cancerstatistics/> (Accessed February 1, 2014).

7. Leber M, Sherma M. Estimation of immigration-related chronic hepatitis B infection and hepatocellular carcinoma development in Canada from 1981-2006. Can J Gastroenterol 2009;23(Suppl A):A22 (Abst).

8. Myers RP, Krajden M, Bilodeau M, et al. Burden of disease and cost of chronic hepatitis C infection in Canada. Can J Gastroenterol Hepatol 2014;28:243-50.

9. Zhang BH, Yang BH, Tang ZY. Randomized controlled trial of screening for hepatocellular carcinoma. J Cancer Res Clin Oncol 20041;130:417-22.

10. Lederle FA, Pocha C. Screening for liver cancer: The rush to judgment. Ann Intern Med 2012;156:387-9.

11. Sharma S, Acarsu U, Hirschfield GM, Feld JJ. Development of a scoring system to predict risk of hepatocellular carcinoma in a cohort of patients with cirrhosis. J Hepatol 2013;58(Suppl 1):S49 (Abst).

12. Yang HI, Yuen MF, Chan HL, et al; REACH-B Working Group. Risk estimation for hepatocellular carcinoma in chronic hepatitis $B$ (REACH-B): Development and validation of a predictive score. Lancet Oncol 2011;12:568-74.

13. Singal A, Volk ML, Waljee A, et al. Meta-analysis: Surveillance with ultrasound for early-stage hepatocellular carcinoma in patients with cirrhosis. Aliment Pharmacol Ther 2009;30:37-47.

14. Singal AG, Marrero JA, Yopp A. Screening process failures for hepatocellular carcinoma. J Natl Compr Canc Netw 2014;12:375-82.

15. Singal AG, Conjeevaram HS, Volk ML, et al. Effectiveness of hepatocellular carcinoma surveillance in patients with cirrhosis. Cancer Epidemiol Biomarkers Prev 2012;21:793-9.

16. Yoon SH, Lee JM, So YH, Hong SH, Kim SJ, Han JK, Choi BI. Multiphasic MDCT enhancement pattern of hepatocellular carcinoma smaller than $3 \mathrm{~cm}$ in diameter: Tumor size and cellular differentiation. AJR Am J Roentgenol 2009;193:482-9.

17. Di Tommaso L, Destro A, Fabbris V, et al. Diagnostic accuracy of clathrin heavy chain staining in a marker panel for the diagnosis of small hepatocellular carcinoma. Hepatology 2011;53:1549-57.

18. Mitchell DG, Bruix J, Sherman M, Sirlin CB. LI-RADS (Liver Imaging Reporting and Data System): Summary, discussion, consensus of the LI-RADS Management Working Group and future directions. Hepatology 2014; doi: 10.1002/hep.27304 [Epub ahead of print].

19. Vilana R, Forner A, Bianchi L, et al. Intrahepatic peripheral cholangiocarcinoma in cirrhosis patients may display a vascular pattern similar to hepatocellular carcinoma on contrast-enhanced ultrasound. Hepatology 2010;51:2020-9.

20. Zheng SG, Xu HX, Liu LN. Management of hepatocellular carcinoma: The role of contrast-enhanced ultrasound. World J Radiol 2014;6:7-14.

21. Jhaveri K, Cleary S, Audet P, et al. A multidisciplinary approach to liver imaging - Consensus statements from a Multidisciplinary Expert Panel on the Utilization and Application of a Liver Specific MR Imaging Contrast Agent (Gd-EOB-DTPA). Am J Roentgenol 2015;204:498-509.

22. Forner A, Llovet JM, Bruix J. Hepatocellular carcinoma. Lancet 2012;379:1245-55.

23. Bolondi L, Burroughs A, Dufour JF, et al. Heterogeneity of patients with intermediate (BCLC B) hepatocellular carcinoma: Proposal for a subclassification to facilitate treatment decisions. Semin Liver Dis 2012;32:348-59.

24. Mazzaferro V, Bhoori S, Sposito C, et al. Milan criteria in liver transplantation for hepatocellular carcinoma: An evidence-based analysis of 15 years of experience. Liver Transpl 2011;(17 Suppl 2):S44-S57.

25. Villanueva A, Hoshida Y, Battiston C, et al. Combining clinical, pathology, and gene expression data to predict recurrence of hepatocellular carcinoma. Gastroenterology 2011;140:1501-12.

26. Llovet JM, Fuster J, Bruix J. Intention-to-treat analysis of surgical treatment for early hepatocellular carcinoma: Resection versus transplantation. Hepatology 1999;30:1434-40.

27. Cucchetti A, Cescon M, Ercolani G, Bigonzi E, Torzilli G, Pinna AD. A comprehensive meta-regression analysis on outcome of anatomic resection versus nonanatomic resection for hepatocellular carcinoma. Ann Surg Oncol 2012;19:3697-705.

28. Cho YK, Kim JK, Kim MY, Rhim H, Han JK. Systematic review of randomized trials for hepatocellular carcinoma treated with percutaneous ablation therapies. Hepatology 2009;49:453-9.

29. Wang Y, Luo Q, Li Y, Deng S, Wei S, Li X. Radiofrequency ablation versus hepatic resection for small hepatocellular carcinomas: A meta-analysis of randomized and nonrandomized controlled trials. PLoS One 2014;9:e84484.

30. Toso C, Meeberg G, Henandez-Alejandro R, et al. Total tumor volume and alpha fetoprotein for selection of transplant candidates with hepatocellular carcinoma: A prospective validation. Hepatology 2015, March 17. (Epub ahead of print).

31. Marelli L, Stigliano R, Triantos C, et al.Transarterial therapy for hepatocellular carcinoma: Which technique is more effective? A systematic review of cohort and randomized studies. Cardiovasc Intervent Radiol 2007;30:6-25.

32. Lammer J, Malagari K, Vogl T, et al; PRECISION V Investigators. Prospective randomized study of doxorubicin-eluting-bead embolization in the treatment of hepatocellular carcinoma: Results of the PRECISION V study. Cardiovasc Intervent Radiol 2010;33:41-52.

33. Burrel M, Reig M, Forner A, et al. Survival of patients with hepatocellular carcinoma treated by transarterial chemoembolisation (TACE) using drug eluting beads. Implications for clinical practice and trial design. J Hepatol 2012;56:1330-5.

34. Llovet JM, Ricci S, Mazzaferro V, et al; SHARP Investigators Study Group. Sorafenib in advanced hepatocellular carcinoma. N Engl J Med 2008;359:378-90.

35. Cheng AL, Kang YK, Chen Z, et al. Efficacy and safety of sorafenib in patients in the Asia-Pacific region with advanced hepatocellular carcinoma: A phase III randomised, double-blind, placebocontrolled trial. Lancet Oncol 2009;10:25-34.

36. Salem R, Mazzaferro V, Sangro B. Yttrium 90 radioembolization for the treatment of hepatocellular carcinoma: Biological lessons, current challenges, and clinical perspectives. Hepatology 2013;58:2188-97.

37. Salem R, Lewandowski RJ, Kulik L, et al. Radioembolization results in longer time-to-progression and reduced toxicity compared with chemoembolization in patients with hepatocellular carcinoma. Gastroenterology 2011;140:497-507.

38. Lewandowski RJ, Kulik LM, Riaz A, et al. A comparative analysis of transarterial downstaging for hepatocellular carcinoma: Chemoembolization versus radioembolization. Am J Transplant 2009;9:1920-8.

39. Klein J, Dawson LA. Hepatocellular carcinoma radiation therapy: Review of evidence and future opportunities. Int J Radiat Oncol Biol Phys 2013;87:22-32.

40. Cheng AL, Kang YK, Lin DY, et al. Sunitinib versus sorafenib in advanced hepatocellular cancer: Results of a randomized phase III trial. J Clin Oncol 2013;31:4067-75.

41. Johnson PJ, Qin S, Park JW, et al. Brivanib versus sorafenib as firstline therapy in patients with unresectable, advanced hepatocellular carcinoma: Results from the randomized phase III BRISK-FL study. J Clin Oncol 2013;31:3517-24.

42. Zhu AX, Rosmorduc O, Evans TR, et al. SEARCH: A phase III, randomized, double-blind, placebo-controlled trial of sorafenib plus erlotinib in patients with advanced hepatocellular carcinoma. J Clin Oncol 2014: pii: JCO.2013.53.7746 (Epub ahead of print).

43. Llovet JM, Decaens T, Raoul JL, et al. Brivanib in patients with advanced hepatocellular carcinoma who were intolerant to sorafenib or for whom sorafenib failed: Results from the randomized phase III BRISK-PS study. J Clin Oncol 2013;31:3509-16.

44. Zhu AX, Kudo M, Assenat E, et al. Effect of everolimus on survival in advanced hepatocellular carcinoma after failure of sorafenib: The EVOLVE-1 randomized clinical trial. JAMA 2014;312:57-67.

45. Shen YC, Lin ZZ, Hsu CH, Hsu C, Shao YY, Cheng AL. Clinical trials in hepatocellular carcinoma: An update. Liver Cancer 2013;2:345-64.

46. Bruix J, Sherman M; Practice Guidelines Committee, American Association for the Study of Liver Diseases. Management of hepatocellular carcinoma. Hepatology 2005;42:1208-36. 


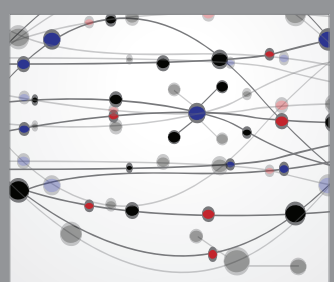

The Scientific World Journal
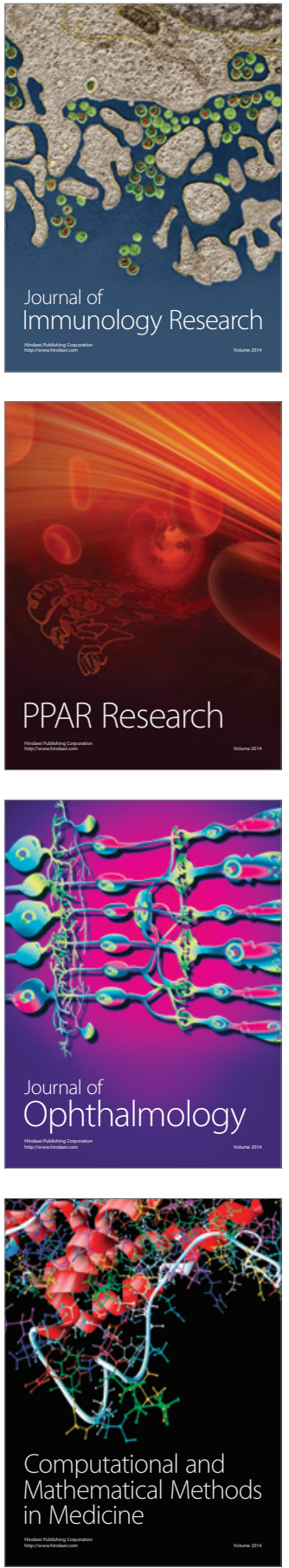

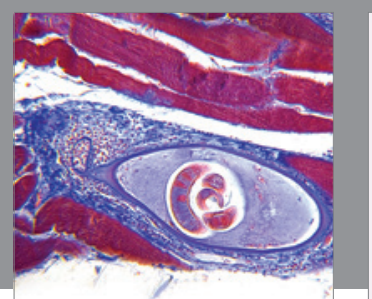

Gastroenterology Research and Practice

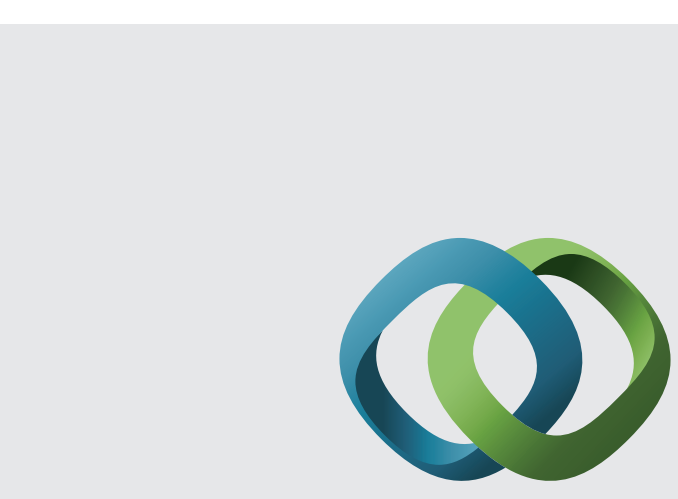

\section{Hindawi}

Submit your manuscripts at

http://www.hindawi.com
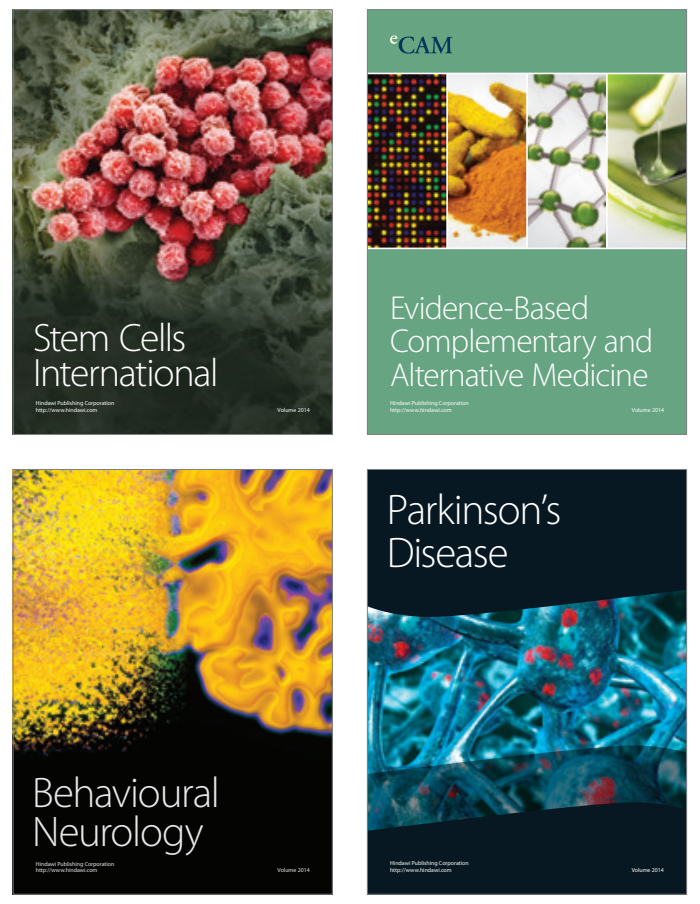
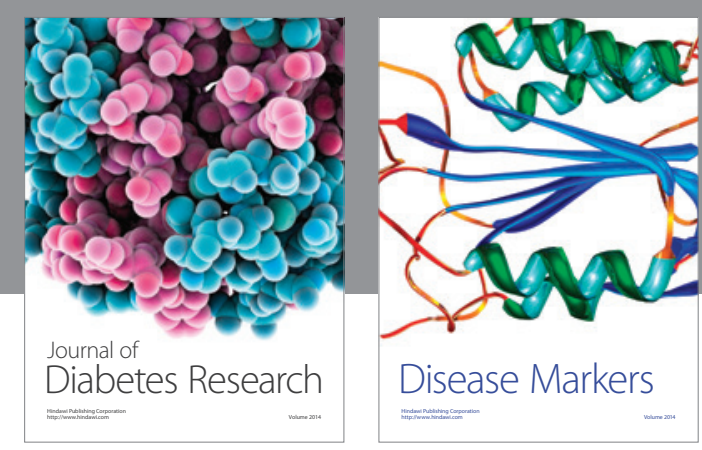

Disease Markers
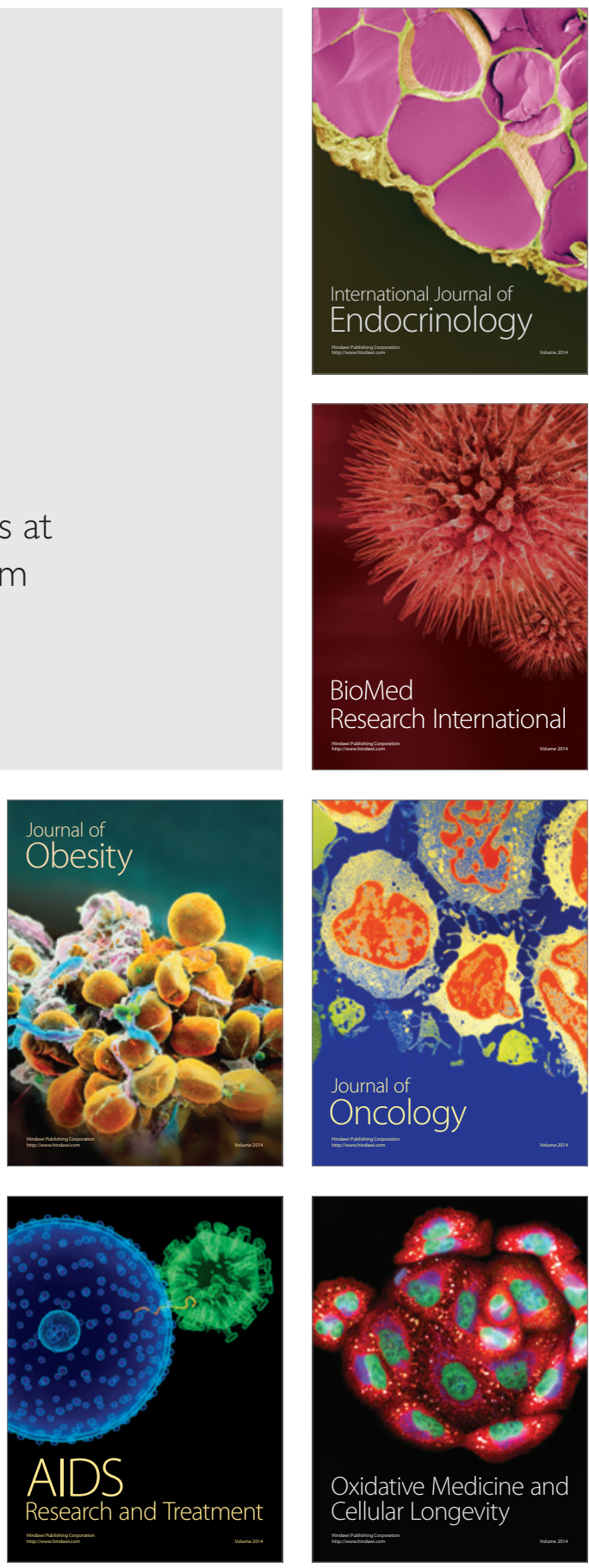